\title{
Spinal Clinic for Obese Out-Patient Project (SCOOP)—A 1 Year Report
}

\author{
Samford Wong ${ }^{1,3}$, Allison Graham ${ }^{2}$, George Grimble ${ }^{3}$, Alastair Forbes ${ }^{3}$ \\ ${ }^{1}$ Department of Nutrition and Dietetics, Stoke Mandeville Hospital, Aylesbury, UK; ${ }^{2}$ National Spinal Injuries Centre, Stoke Mande- \\ ville Hospital Aylesbury, UK; ${ }^{3}$ Centre for Gastroenterology and Clinical Nutrition, University College London, London, UK. \\ Email: Samford.Wong@ucl.ac.uk
}

Received July 26 ${ }^{\text {th }}, 2011$; revised August 22 ${ }^{\text {nd }}, 2011$; accepted August $29^{\text {th }}, 2011$.

\begin{abstract}
Patients with spinal cord injury (SCI) appear to be at higher risk of becoming overweight after their injury. This 12 month study aimed to assess the effectiveness of a dietitian-led clinic. Thirty-eight patients with chronic SCI with a body mass index (BMI) range of $26.4-46.4 \mathrm{~kg} / \mathrm{m}^{2}$ were referred for three consultations over a three month period for dietetic advice covering nutrition, exercise and behaviour change. Body composition was estimated by anthropometric measurements of BMI, mid upper-arm circumference (MUAC), triceps-skinfold thickness (TSF), mid-arm muscle circumference (MAMC) and sitting blood pressure. Nineteen individuals completed the three month intervention. There were significant reductions in weight ( $\mathrm{kg}: 103.1 v$ 97.8, $P<0.001), B M I\left(\mathrm{~kg} / \mathrm{m}^{2}\right.$ : $\left.35.5 v 34.0, P<0.001\right), T S F$ (mm: $28.3 v 24.7$, $P=0.019)$, and sitting systolic blood pressure ( $\mathrm{mm} \mathrm{Hg:} 134 v$ 101, $P=0.015)$, and an increase in MAMC (cm: $29.5 v$ $30.0, P=0.045)$. We conclude that a simple dietetic intervention can help individuals with SCI to lose weight without compromising lean body mass. Although the intervention incurred additional cost, it has the potential to decrease long-term healthcare expenditure if patients' outcome and quality of life are improved.
\end{abstract}

Keywords: Obesity, Malnutrition, Nutritional Intervention, Spinal Cord Injury

\section{Introduction}

The management of obesity in individuals with spinal cord injury (SCI) is important because it is highly prevalent ( $74 \%$ of this population in one study) [1] but there is little evidence to suggest that its management can be effective. This contrasts to some extent with the situation for the general population in the UK, where one in four adults is now obese and treatment protocols as well established. But in both scenarios the prevalence can be expected to increase [2], with serious health and cost implications [2,3]. There are limited data on the rate of weight gain after SCI in the UK, but in comparison with the UK national survey data [4], patients with SCI [1] seem more likely to become overweight and obese than the able-bodied population ( $74 \%$ vs. $40 \%$ ).

Apart from the obesogenic environment, SCI patients are more vulnerable to overnutrition because of their inherent immobility and muscle atrophy due to paralysis and subsequent changes in body composition. This may arise because the combination of a lower basal metabolic rate [5] and restricted physical activity [6] result in an especially positive energy balance and therefore weight gain.
The consequences of obesity include an increased risk of premature death and the risk of developing chronic diseases, such as type II diabetes mellitus, cardiovascular disease (CVD), sleep apnoea, gallstones and certain forms of cancer. The risk of mortality associated with CVD is twice as high in SCI patients compared with able-bodied individuals with CVD [7]. Indeed, for long-term SCI individuals, morbidity and mortality from CVD have now exceeded those caused by renal and pulmonary conditions and CVD has become the primary cause of death [7]. In addition, overweight SCI patients are at a higher risk of developing pressure ulcers [8], increasing the difficulties in managing daily life with SCI [9].

Many of the conditions related to obesity could be managed or prevented by appropriate nutritional interventions. In 2006, the National Institute for Health and Clinical Excellence (NICE) published guidelines on managing overweight and obesity [3]. Although weight loss has been advocated as a primary treatment strategy for the condition, to date little high quality evidence exists to support this concept in patients with SCI. There are limited published data on the effect of dietary intervention in obese SCI individuals $[10,11]$ and no formal approach 
has been tried in the British SCI centres. This study therefore aimed to investigate the effectiveness of a dietitian led weight management clinic in a tertiary spinal injuries centre in the UK.

\section{Subject and Methods}

\subsection{Study Design}

The study was a single group, uncontrolled study conducted at the National Spinal Injuries Centre, Stoke Mandeville Hospital, Aylesbury, UK over 1 year period from September 2008 to September 2009.

\subsection{Subjects}

Potential patients were screened by out-patient staff when they attended the department for routine health reviews. Those eligible for inclusion were individuals with SCI, who were adult (aged 18 years or over) and with a BMI > $28 \mathrm{~kg} / \mathrm{m}^{2}$. Eligible patients were offered the opportunity to attend the pilot weight management clinic by their consultants. Patients were followed up on 2 occasions after the initial appointment (week 0), at week 4 and week 12. Data were recorded at baseline and repeated when participants attended their follow-up appointments by the same researcher.

Data were collected following a standardised protocol, which included demographic information, sex, age, and ethnicity, anthropometric measurements, sitting blood pressure, location and completeness of SCI. A brief personal and family history and past medical history were also collected.

\subsection{Anthropometric Measurements}

Each anthropometric measurement was performed twice by the same researcher who was fully trained in anthropometry. An average of the two measurements was taken. If the measurement difference was greater than $10 \%$, a third measurement was performed with the aim of increasing accuracy.

Participants were weighed using a calibrated digital wheelchair scale (Avery Berkley L115, UK). Two measurements were taken, one of the participant and the wheelchair, and the other of the empty wheelchair; the participant's weight was then calculated as the difference between these measurements. Height was collected by recalled height or estimated by knee height callipers [12, 13]. (Harpenden Anthropometer, Crymych, Pembrokeshire, UK) BMI was calculated from height, and weight. Mid upper arm circumference (MUAC) of the left arm was measured in addition to triceps skin fold thickness (TSF) using callipers (Holtain, Holtain Ltd, Crymych, London, UK) in order to obtain an estimate of mid-arm muscle circumference (MAMC) as described before [14].

\subsection{Life Style Modification}

At each appointment, goals were set, with the dietitian and the patient usually agreeing on two targets for the patient to achieve for the next appointment. General healthy dietary advice based on the eat-well plate was discussed in the initial appointment. Goal setting on behavioural change around dietary intake was based on the local weight management policy in line with the NICE guideline [3]. Encouragement in improving physical activity—such as pushing their wheelchair for up to 30 minutes five times per week (over and above normal daily movements) — was recommended to those who had upper limb power.

A protocol, developed by Foreyt and colleagues [15] was used to help patients to introduce lifestyle changes. After the initial appointment, patients were provided with the dietitian's contact number in case further advice and/ or resolution of any queries were needed. On the subsequent follow up, if patients had not achieved their original goals, new goals were not discussed (Figure 1).

\subsection{Statistical Methods}

All the data (checked for normality) are expressed as mean values with standard deviation (s.d). Changes were calculated by subtracting the baseline measurements from the 12 week follow-up measurements. A paired t-test was used to determine the significance of differences in changes from baseline with a statistical significance considered present at the $5 \%$ level $(P=0.05)$. All analysis was done on an intention to treat basis, with baseline data carried forward for all missing data. All statistical tests were conducted using the Minitab Statistical Software (Version 15.0, Minitab Inc).

\subsection{Ethics}

After consultation with the research board and the management board in the National Spinal Injuries Centre, this study was considered a service evaluation/audit, and therefore did not require formal ethical approval, but the patients were still informed about the study and verbal consent was obtained.

\section{Results}

Thirty-eight patients were seen between September 2008 and September 2009 (Figure 1), 27 were subsequently offered follow-up appointments, and 19 of these completed the 12 week programme and were included in this analysis.

\section{Family history and co-morbidity}

Eighteen patients (66\%) reported a family history of nutritional related complications, 16 (59.3\%) had at least one co-morbidity; 8 (29.6\%) were current smokers and two $(7.4 \%)$ were ex smokers; 11 (40.7\%) consumed alcoholic beverages regularly.

\section{Weight gain since $S C I$}

The average weight gain after SCI of the 38 patients with known pre-injury weight was $7.6 \mathrm{~kg}(\mathrm{SD} \pm 4.2 \mathrm{~kg})$. It was 


\begin{tabular}{|c|c|c|c|}
\hline Week & Time & & Aims of appointment \\
\hline 0 (Initial appointment): & $60 \mathrm{mins}$ & & $\begin{array}{l}\text { Introduction to weight management, } \\
\text { Anthropometric measurements } \\
\text { (weight, height, MUAC, TSF) } \\
\text { Nutritional assessment } \\
\text { Dietary, activity and weight target goal setting } \\
\text { Set } 2-3 \text { small goals for next appointment }\end{array}$ \\
\hline 4 & 30 mins & & $\begin{array}{l}\text { Repeat anthropometric measurements } \\
\text { Reviewing target set at week } 0 \\
\text { Discuss diet history / eating pattem where } \\
\text { appropriate } \\
\text { Encourage exercise if appropriate } \\
\text { Give individualise diet sheet if appropriate } \\
\text { Discuss and agree goals for next appointment }\end{array}$ \\
\hline 8 & $30 \mathrm{mins}$ & & $\begin{array}{l}\text { Repeat anthropometric measurements } \\
\text { Review target set at week } 4 \\
\text { Discuss target achieved / not achieved } \\
\text { Discuss reasons why if not achieved } \\
\text { If weight loss target achieved, discuss weight } \\
\text { maintenance } \\
\text { Discuss and agree goals for next appointment }\end{array}$ \\
\hline 12 & 30 mins & - & $\begin{array}{l}\text { Repeat anthropometric measurements } \\
\text { Review target set } \\
\text { If weight loss achieved, discuss / encourage } \\
\text { weight maintenance } \\
\text { Discuss the need for any further follow up } \\
\text { Options include } \\
\text { Discharge } \\
\text { Six / annual follow up } \\
\text { Weight loss support group outside SCI centre }\end{array}$ \\
\hline 36 & $30 \mathrm{mins}$ & & Six month follow up if appropriate \\
\hline
\end{tabular}

Figure 1. Clinic protocol.

estimated that they were gaining an average of $2.5 \mathrm{~kg}$ per year.

Eleven patients were excluded from the analysis due to advanced age [2 (3.6\%)], prominent co-morbidity [3 (5.5\%)] and initial BMI $<28 \mathrm{~kg} / \mathrm{m}^{2}$ [6 (11.1\%)] (Figure 2). The patients' characteristics and their demographic, an- thropometric, age and gender specific values are summa- rised in Tables 1 and 2.

Vitamin supplement consumption was reported by $6 / 22$ (27.3\%) participants.

Drop-outs and adverse effects of the programme

Eight patients dropped out after their initial appointment, and six more before the 12-week follow-up. The entire 12 week programme was completed by 7 of 8 (87.5\%) women and 12 of 19 (63.5\%) men. The drop-out rate did not differ significantly between sex $(P=0.21)$, or the stage of the program $(P=0.10)$.

We categorised the distance from each patient's registered address to the tertiary centre into 3: (<20 miles, 20 50 miles and $>50$ miles) and compared attendance rates. No statistical difference was found between initial $(<20$ miles: 63.6\%; 20 - 50 miles: 68\%; $>50$ miles: 73.6\%, $\chi^{2}: P=$
0.387), follow-up (<20 miles: 70\%, 20 - 50 miles: 86.4\%; $>50$ miles: $76.2 \%, x^{2}: P=0.456$ ) or and combined initial and follow-up appointment rates (<20 miles: 66.7\%; 20 - 50 miles: 78.8\%; $>50$ miles: $\left.75 \%, x^{2}: P=0.527\right)$.

Changes after 12 weeks in the programme

Body weight, subcutaneous fat and sitting blood pressure were significantly reduced whilst arm muscle increased. The average weight loss of patients at week 12 was 3.7 $\mathrm{kg} \pm$ (SD: $3.1 \mathrm{~kg}$ ) (Table 2). The weight loss was from (mean (SD)) $103.1 \mathrm{~kg}$ (19.7) to $97.8 \mathrm{~kg}(18.2 ; P<0.0001)$, and the BMI from $35.5 \mathrm{~kg} / \mathrm{m}^{2}$ (5.6) to $34.0 \mathrm{~kg} / \mathrm{m}^{2}$ (5.6; $P<$ $0.0001)$ at 12 -weeks. There was a statistically significant change in TSF from $28.3 \mathrm{~mm}$ (7.18) to $24.7 \mathrm{~mm}$ (5.81; $P$ $=0.019$ ), and in MAMC from $29.5 \mathrm{~cm}$ (4.98) to $30.0 \mathrm{~cm}$ (5.69; $P=0.045)$, but no difference was observed in MUAC (38.5 cm vs. $39.3 \mathrm{~cm})$. In addition, sitting systolic blood pressure $(\mathrm{mm} / \mathrm{Hg})$ had fallen from 134 (16.9) to 101 (36.9; $P=0.026$ ); the diastolic pressure was unchanged (88 vs. 86.9) (Table 3 ).

\section{Discussions}

The most important therapeutic finding was that dietary 


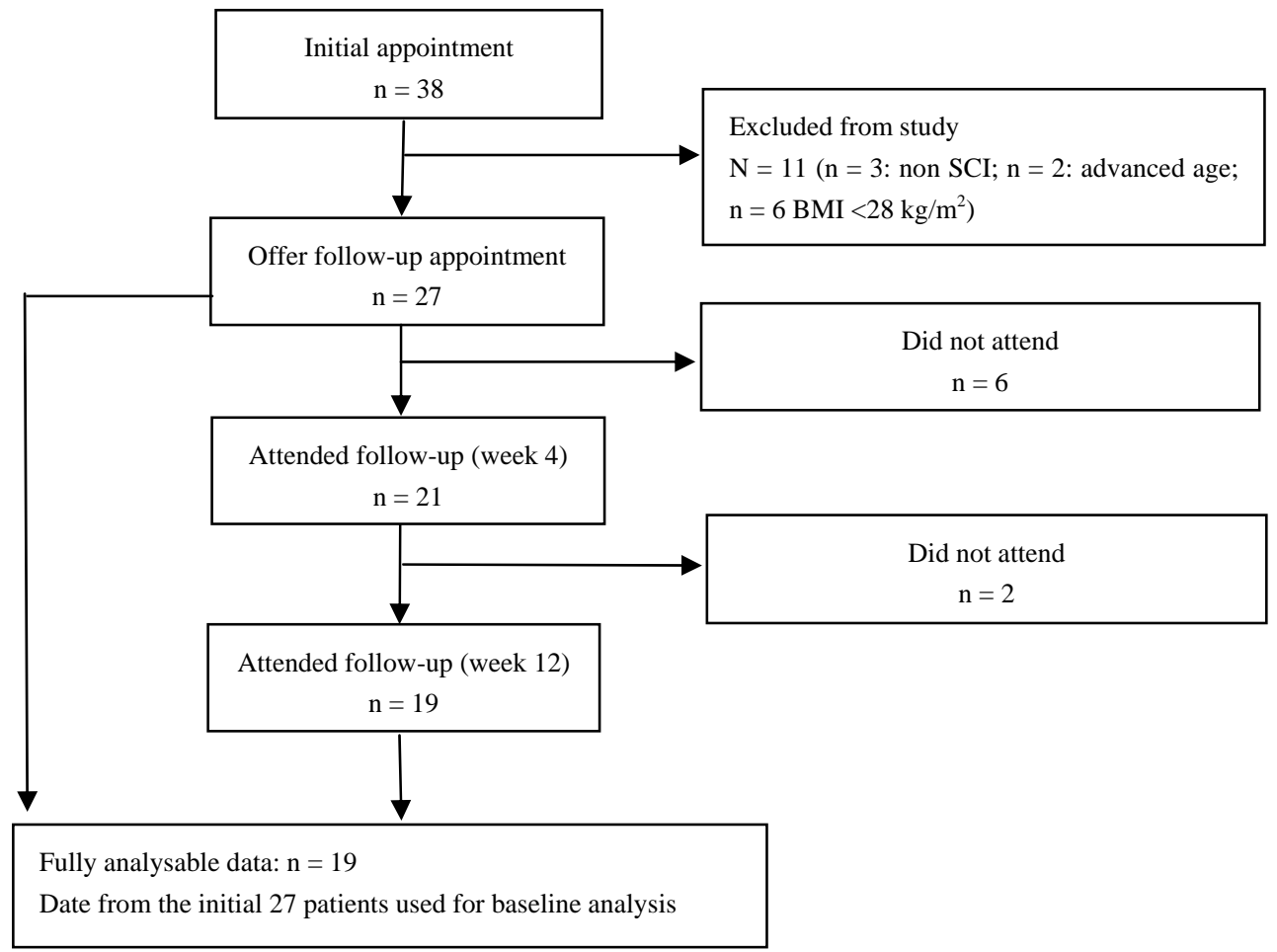

Figure 2. Clinic flowchart.

Table 1. Summary of the baseline data (anthropometric indices).

\begin{tabular}{|c|c|c|c|c|c|c|c|c|}
\hline \multirow[b]{2}{*}{ Variables } & \multirow[b]{2}{*}{$\mathrm{n}$} & \multirow[b]{2}{*}{ Mean } & \multirow[b]{2}{*}{ SD } & \multicolumn{5}{|c|}{ Range } \\
\hline & & & & Minimum & Q1 & Median & Q3 & Maximum \\
\hline Age (Years) $(P=0.649)$ & 27 & 47.1 & 13.42 & 24 & 37 & 44.0 & 53 & 75 \\
\hline Male: & 19 & 45 & 14.7 & 24 & 37 & 45.0 & 53 & 75 \\
\hline Female: & 8 & 42 & 10.32 & 36 & 37.5 & 42.0 & 53.8 & 65 \\
\hline Weight $(\mathrm{Kg}) *(P=0.038)$ & 27 & 103.1 & 3.81 & 70.3 & 91.3 & 103 & 113.8 & 154.8 \\
\hline Male: & 19 & 108.2 & 19.32 & 71.9 & 98.9 & 104.9 & 122.6 & 154.8 \\
\hline Female: & 8 & 91.1 & 16.1 & 70.3 & 75.9 & 91.8 & 105.8 & 116.2 \\
\hline Height $(\mathrm{cm})(P=0.202)$ & 27 & 1.70 & 0.14 & 1.27 & 1.65 & 1.70 & 1.83 & 1.85 \\
\hline Male: & 19 & 1.73 & 0.16 & 1.27 & 1.67 & 1.8 & 1.83 & 1.85 \\
\hline Female: & 8 & 1.65 & 0.05 & 1.54 & 1.63 & 1.65 & 1.69 & 1.70 \\
\hline $\operatorname{BMI}\left(\mathrm{Kg} / \mathrm{m}^{2}\right)(P=0.243)$ & 27 & 35.51 & 5.63 & 26.4 & 30.5 & 34.2 & 40.1 & 46.4 \\
\hline Male: & 19 & 36.34 & 5.1 & 28.7 & 32.1 & 36.6 & 40.2 & 45 \\
\hline Female: & 8 & 33.5 & 5.06 & 26.4 & 27.8 & 32.1 & 38.6 & 46.4 \\
\hline $\operatorname{MUAC}(\mathrm{cm})(P=0.122)$ & 24 & 38.48 & 4.54 & 29.2 & 34.8 & 38.7 & 41.2 & 46.7 \\
\hline Male: & 17 & 39.4 & 3.66 & 34.2 & 35.9 & 39.0 & 41.2 & 46.7 \\
\hline Female: & 7 & 36.23 & 5.93 & 29.2 & 31.8 & 34.7 & 42 & 45.6 \\
\hline $\operatorname{TSF}(\mathrm{mm})(P=0.394)$ & 24 & 28.32 & 7.18 & 15.0 & 25.1 & 29 & 30.8 & 45.7 \\
\hline Male: & 17 & 27.5 & 8.07 & 15 & 20.6 & 27.8 & 30.3 & 45.7 \\
\hline Female: & 7 & 30.31 & 4.16 & 25.0 & 26.2 & 30.0 & 35.0 & 36.2 \\
\hline $\operatorname{MAMC}(\mathrm{cm})(P=0.074)$ & 23 & 29.53 & 4.98 & 21.3 & 25.8 & 29.1 & 31.6 & 41.8 \\
\hline Male: & 16 & 30.75 & 4.53 & 23.4 & 28.2 & 30.8 & 33.0 & 41.8 \\
\hline Female: & 7 & 26.73 & 5.14 & 21.3 & 22.4 & 25.8 & 31.0 & 35.9 \\
\hline
\end{tabular}

BMI: Body Mass Index; MUAC: Mid upper arm circumference; TSF: Triceps skinfold thickness; MAMC: Mid arm muscle circumference $* P<0.05$ (two sample t-test). 
Table 2. Patient characteristics.

\begin{tabular}{ccc}
\hline & $\mathrm{N}$ & Percentage (\%) \\
\hline Level of SC & 13 & 44.8 \\
Cervical & 4 & 13.8 \\
Lumbar & 11 & 37.9 \\
Thoracic & & \\
AIS $\quad 11$ & 37.9 \\
A & 2 & 6.9 \\
B & 9 & 31.1 \\
C & 7 & 24.1 \\
D & & \\
Ethnic origin & 21 & 77.7 \\
Caucasian & 5 & 18.6 \\
Asian & 1 & 3.7 \\
Afro-Caribbean & &
\end{tabular}

AIS: American Spinal Injury Association Impairment Scale; CI: Spinal cord injuries.
Intervention is associated with a clinically meaningful reduction in weight in overweight SCI individuals. Overall weight was reduced by $3.5 \%$ in this study in line with the results of the single previous study (3.8\%) [11].

It is tempting to conclude that weight gain was simply a consequence of overnutrition, reduced physical activity or a combination of both. It is certainly likely that SCI individuals would have been less physically active [6], indicating a lower energy need to maintain the total energy expenditure, which is compatible with lower energy intake. But it has also been suggested that there is an important change in body composition after SCI, with reduced lean body mass and increased fat mass [7] leading to reduced nutritional needs [6]. In the clinical setting, regular education of all SCI individuals regarding reduced nutritional requirements may help to prevent excess weight gain. Additional assessment or interventions may still be required for overweight SCI patients.

Eight people did not attend the follow-up clinic. The drop-out rate could not be explained by the distance that patients had to travel in order to attend. Instead, it suggests that a lack of readiness for change by the individuals may be a more important reason for non-attendance [3].

Table 3. Summary of post-clinic changes.

\begin{tabular}{|c|c|c|c|c|c|}
\hline & $\mathrm{n}$ & Initial & Final & Change & $P$-value \\
\hline \multicolumn{6}{|l|}{ Variable } \\
\hline & & mean (s.d.) & mean (s.d.) & mean (s.d.) & \\
\hline Weight (kg) $* *$ & 19 & $103.1(19.77)$ & $97.8(18.2)$ & $3.7(3.1)$ & 0.0001 \\
\hline \% weight loss (Range: $0.82 \%$ to $12 \%$ ) & & & & $3.5 \%$ & \\
\hline $\operatorname{BMI}\left(\mathrm{kg} / \mathrm{m}^{2}\right) * *$ & 19 & $35.5(5.63)$ & $34.0(5.6)$ & $1.28(1.0)$ & 0.0001 \\
\hline \multicolumn{6}{|l|}{ Anthropometric measurements } \\
\hline MUAC (cm) & 18 & $38.5(4.54)$ & $39.3(4.29)$ & $0.185(1.49)$ & 0.66 \\
\hline TSF (mm)* & 18 & $28.3(7.18)$ & $24.7(5.81)$ & $2.53(4.12)$ & 0.019 \\
\hline MAMC $(\mathrm{cm})^{*}$ & 18 & $29.5(4.98)$ & $30.0(5.69)$ & $0.729(1.385)$ & 0.045 \\
\hline \multicolumn{6}{|l|}{ Sitting blood pressure } \\
\hline Systolic $(\mathrm{mm} / \mathrm{Hg}) *$ & 19 & 134 (16.9) & $101(36.9)$ & $33.1(25.8)$ & 0.015 \\
\hline Diastolic (mm/Hg) & 19 & $88(25.8)$ & $86(27.0)$ & $20.9(38.9)$ & 0.206 \\
\hline
\end{tabular}

BMI, Body Mass Index; MUAC, mid upper arm circumference; TSF, triceps skinfold; MAMC, mid arm muscle circumference; *Statistically significant differences in mean values between pre and post 12 week consultations $(P<0.05)$; **Statistically significant differences in mean values between pre and post 12 week consultations $(P<0.01)$. 
A strength of this study is that it is the first reported clinical attempt to treat obesity in SCI in the UK. The intervention includes the use of low cost dietary intervention delivered by a dietitian and avoids the higher costs and potential side effects caused by drug intervention or bariatric surgery. Although bariatric surgery, which is regarded as the most effective method for treating obesity, and can result in sustained weight loss of $25 \mathrm{~kg}$ - $30 \mathrm{~kg}$ [16], the rising prevalence of obesity will preclude its provision to all patients with this problem, and especially to those with other major problems such as SCI. Non-surgical treatment options, preferably of lower cost and with proved effectiveness are therefore needed. All of the data collected in this study were readily available as routine clinical information, permitting additional savings, as expensive equipment for sophisticated tests and monitoring is not needed.

However, the study has limitations. For example, this opportunistic sample of patients was not compared with a control group. A dropout rate of $29.6 \%$ (8/27), limited the statistical power of the study; the follow-up period was relatively short, and long-term adherence to the weight management programme was not determined. Most programmes would follow patients for at least a year but, encouragingly if anecdotally, one of our patients has maintained weight loss after 16 months [17].

This study included only those with a BMI $>28 \mathrm{~kg} / \mathrm{m}^{2}$, and it is possible that resources expended on those less severely overweight might be more cost-effective. The use of sophisticated equipment such as dual-energy x-ray absorptiometry, would have provided more accurate body fat and lean body mass information, and this will be important in the future determination of the mechanisms for obesity in SCI [18].

All the patients in this study were weighed using a wheelchair scale. Due to their inability to stand, their height was estimated by alternative surrogate measurements $[12,13]$. Experience from clinical practice suggests it is unrealistic to expect height to be measured by general clinic staff. When requested it is frequently not carried out because of work pressures and limited availability of equipment. Similarly waist circumference-which can be an alternative measurement of body adiposity [19]—is not measured routinely, and attempts to get it done are often unsuccessful (Graham A: personal communication). All of these, and the use of skinfold callipers, can however be employed as useful bedside clinical tools to assess body fat, and body muscle mass once the patient has come to specialist dietetic attention and are accordingly advocated [18].

Recently, adjusted BMI limits of 22 and $27 \mathrm{~kg} / \mathrm{m}^{2}$ have been proposed as cut offs for designation of overweight and obesity in SCI individuals [20]. Despite the fact that researchers in SCI medicine have argued that BMI may not be appropriate for SCI patients, at present national guidelines retain the universal reference values of 25 for overweight and 30 for obesity [3], and BMI remains an important anthropometric index for evaluation of the risk of disease associated with obesity [3]. This will undoubtedly be the subject of future research effort and whether there might be better simple surrogate measures to estimate adiposity in SCI.

In conclusion, weight gain after SCI is common. This is most likely due to a combination of reduced nutritional requirements secondary to enforced inactivity and immobilization due to paralysis and a change in the body composition. The present study has shown that overnutrition is a major clinical problem in patients with chronic SCI. We consider that there is an unmet need to define nutritional requirements in SCI patients and to improve the methods for screening. Nonetheless it appears that a successful weight management programme can be run within a SCI Centre and that, along with weight loss, it is possible for individuals to maintain total lean body mass.

On the basis of this study, the out-patient department at Stoke Mandeville has instituted a care pathway to screen and refer patients for weight management. Although this has incurred increased cost, it has been considered worthwhile through its potential to decrease ultimate health care costs by improving the patient's clinical outcome and quality of life [11]. This was however a pilot study and the results indicate the need for further, larger-scale investigations to confirm the prevalence of overnutrition and the quantitative benefits of this approach.

\section{Acknowledgements}

The authors would like to express their appreciation for the efforts of the Outpatient Department staff at the National Spinal Injuries Centre.

The authors are pleased to acknowledge their support from the UK National Institute for Health Research (NIHR) via its funding of the UCL/UCLH Comprehensive Biomedical Research Centre.

\section{Conflict of Interest}

Part of the study were presented at the Nutrition Society annual meeting in July 2010 at Heriot-Watt University, Edinburgh, UK and the European Society of Parenteral and Enteral Nutrition annual meeting in September 2010. This project was partially funded by an unrestricted educational grant from Sanofi-Aventis: NPR 08/1154

\section{Contributions}

SW-Clinic development, clinical measurements, data analysis, manuscript preparation

AG_-clinic development, clinical supervision, manuscript revision

GKG-Academic supervision, manuscript revision 
AF-Academic supervision, manuscript revision

\section{REFERENCES}

[1] S. L. Groah, M. S. Nash, I. H. Ljungberg, A. Libin, L. F. Hamm, E. Ward, P. A. Burns and G. Enfield, "Nutrient Intake and Body Habits after Spinal Cord Injury: An Analysis by Sex and Level of Injury," Journal of Spinal Cord Medicine, Vol. 32, No. 1, 2009, pp. 25-33.

[2] Government Office for Science, "Foresight: Tackling Obesities: Furture Choices-Project Report,” HMSO, London, 2007.

[3] NICE "Guidance on the Prevention, Identification, Assessment and Management of Overweight and Obesity in Adults and Children,” NICE, London, 2006. http://www.nice.org.uk/guidance/index.jsp?action=folder $\& 0=47557$

[4] L. Henderson, J. Gregory, K. Irving and G. Swan “The National Diet and Nutritional Survey: Adults Aged 1964 Years. Volume 2: Energy, Protein, Carbohydrates, Fat and Alcohol Intake,” HMSO, London, 2003.

[5] A. C. Buchholz and P. B. Pencharz "Energy Expenditure in Chronic Spinal Cord Injury," Current Opinion in Clinical Nutrition \& Metabolic Care, Vol. 7, No. 6, 2004, pp. 635-639. doi:10.1097/00075197-200411000-00008

[6] A. C. Buchholz, C. F. McGillvray and P. B. Pencharz, "Physical Activity Levels Are Low in Free-Living Adults with Chronic Paraplegia,” Obesity Research, Vol. 11, 2003, pp. 563-570. doi:10.1038/oby.2003.79

[7] J. Myers, M. Lee and J. Kiratli, "Cardiovascular Disease in Spinal Cord Injury: An Overview of Prevalence, Risk, Evaluation and Management," American Journal of Physical Medicine \& Rehabilitation, Vol. 86, No. 2, 2007, pp. 142152. doi:10.1097/PHM.0b013e31802f0247

[8] J. J. Elsner and A. Gefen, "Is Obesity a Risk Factor for Deep Tissue Injury in Patients with Spinal Cord Injury?” Journal of Biomechanics, Vol. 41, No. 16, 2008, pp. 3322-3331. doi:10.1016/j.jbiomech.2008.09.036

[9] R. T. Abresch, D. A. McDonald, L. M. Widman, K. McGinnis and K. J. Hickey, "Impact of Spinal Cord Dysfunction and Obesity on the Health-Related Quality of Life of Children and Adolescents," Journal of Spinal Cord Medicine, Vol. 30, No. S1, 2007, pp. S112-S118.

[10] E. D. Zemper, D. G. Tate, S. Roller, M. Forchheimer, A. Chiodo, V. S. Nelson and W. Scelza, "Assessment of a Holistic Wellness Program for Persons with Spinal Cord Injury,” American Journal of Physical Medicine \& Rehabilitation,
Vol. 82, No. 12, 2003, pp. 957-968. doi:10.1097/01.PHM.0000098504.78524.E2

[11] Y. Chen, S. Henson, A. B. Jackson and J. S. Richards, "Obesity Intervention in Persons with Spinal Cord Injury,” Spinal Cord, Vol. 44, No. 2, 2006, pp. 82-91. doi:10.1038/sj.sc.3101818

[12] W. C. Chumlea and S. Guo, "Equations for Predicting Stature in White and Back Elderly Individuals,” Journals of Gerontology, Vol. 47, 1992, pp. M197-M203.

[13] M. Elia "Screening for Malnutrition: A Multidisciplinary Responsibility. Development and Use of the Malnutrition Universal Screening Tool (MUST) for Adults,” BAPEN, 2003. www.bapen.co.uk

[14] C. W. Bishop, P. E. Bowen and S. I. Ritchley, "Norms for Nutritional Assessment of American Adults by Upper Arm Anthropometry," American Journal of Clinical Nutrition, Vol. 34, No. 11, 1981, pp. 2530-2539.

[15] J. P. Foreyt, "Need for Lifestyle Intervention: How to Begin,” American Journal of Cardiology, Vol. 96, No. 4, 2005, pp. 11E-14E. doi:10.1016/j.amjcard.2005.05.009

[16] L. Sjostrom, A. K. Lindroos, M. Peltonen, et al., "Life Style, Diabetes and Cardiovascular Risk Factors 10 Years after Bariatric Surgery," New England Journal of Medicine, Vol. 351, 2004, pp. 2683-2693. doi:10.1056/NEJMoa035622

[17] S. S. Wong, A. Graham, A. Forbes and G. Grimble, "Weight Management of a Patient with Fourth Cervical and Fifth Cervical Vertebrae Spinal Cord Injury: A Case Study,” Proceedings of the Nutrition Society, Vol. 68, 2009, p. E95. doi:10.1017/S0029665109990486

[18] M. Miller, W. K. Wong, J. Wu, S. Cavenett, L. Daniels and M. Crotty, "Upper-Arm Anthropometry: An Alternative Indicators of Nutritional Health to Body Mass Index in Unilateral Lower-Extremity Amputees?” Archives of Physical Medicine and Rehabilitation, Vol. 89, No. 10, 2008, pp. 2031-2033. doi:10.1016/j.apmr.2008.03.025

[19] A. C. Buchholz and J. M. Bugaresti, "A Review of Body Mass Index and Wasit Circumference as Markers of Obesity and Coronary Heart Disease Risk in Persons with Chronic Spinal Cord Injury,” Spinal Cord, Vol. 43, No. 9, 2005, pp. 513-518. doi:10.1038/sj.sc.3101744

[20] G. E. Laughton, A. C. Buchholz, K. A. M. Ginis and R. E. Goy, "Lowering Body Mass Index Cutoffs Better Identifies Obese Persons with Spinal Cord Injury,” Spinal Cord Vol. 47, No. 10, 2009, pp. 757-762. doi:10.1038/sc.2009.33 